\title{
Dependency and AMR Embeddings for Drug-Drug Interaction Extraction from Biomedical Literature
}

\author{
Yanshan Wang \\ Mayo Clinic \\ 2001 st ST SW \\ Rochester, MN 55902 \\ wang.yanshan@mayo.edu \\ Liwei Wang \\ Mayo Clinic \\ 200 1st ST SW \\ Rochester, MN 55902 \\ wang.liwei@mayo.edu
}

\author{
Sijia Liu \\ Mayo Clinic \\ 2001 st ST SW \\ Rochester, MN 55902 \\ liu.sijia@mayo.edu \\ Feichen Shen \\ Mayo Clinic \\ 200 1st ST SW \\ Rochester, MN 55902 \\ shen.feichen@mayo.edu
Hongfang Liu*
Mayo Clinic
200 1st ST SW
Rochester, MN 55902 \\ liu.hongfang@mayo.edu
}

\author{
Majid Rastegar-Mojarad \\ Mayo Clinic \\ 200 1st ST SW \\ Rochester, MN 55902 \\ mojarad.majid@mayo.edu \\ Fei Liu \\ University of Central Florida \\ 4000 Central Florida Blvd \\ Orlando, FL 32816 \\ feiliu@cs.ucf.edu
}

\begin{abstract}
Drug-drug interaction (DDI) is an unexpected change in a drug's effect on the human body when the drug and a second drug are co-prescribed and taken together. As many DDIs are frequently reported in biomedical literature, it is important to mine DDI information from literature to keep DDI knowledge up to date. One of the SemEval challenges in the year 2011 and 2013 was designed to tackle the task where the best system achieved an F1 score of 0.80 . In this paper, we propose to utilize dependency embeddings and $\mathrm{Ab}-$ stract Meaning Representation (AMR) embeddings as features for extracting DDIs. Our contribution is two-fold. First, we employed dependency embeddings, previously shown effective for sentence classification, for DDI extraction. The dependency embeddings incorporated structural syntactic contexts into the embeddings, which were not present in the conventional word embeddings. Second, we proposed a novel syntactic embedding approach using AMR. AMR aims to abstract away from syntactic idiosyncrasies and attempts to capture only the core meaning of a sentence, which could potentially improve DDI extraction from sentences. Two classifiers (Support Vector Machine and Random Forest) taking these embedding features as input were evaluated on the DDIExtraction 2013 challenge corpus. The experimental results show the effectiveness of dependency and AMR embeddings in the DDI extraction task.
\end{abstract}

${ }^{*}$ Corresponding author.

Permission to make digital or hard copies of all or part of this work for personal or classroom use is granted without fee provided that copies are not made or distributed for profit or commercial advantage and that copies bear this notice and the full citation on the first page. Copyrights for components of this work owned by others than ACM must be honored. Abstracting with credit is permitted. To copy otherwise, or republish, to post on servers or to redistribute to lists, requires prior specific permission and/or a fee. Request permissions from permissions@acm.org.

ACM-BCB'17, August 20-23, 2017, Boston, MA, USA.

(C) 2017 ACM. ISBN 978-1-4503-4722-8/17/08 .. \$15.00

DOI: http://dx.doi.org/10.1145/3107411.3107426
The best performance was obtained by combining word, dependency and AMR embeddings (F1 score $=0.84$ ).

\section{CCS CONCEPTS}

- Computing methodologies $\rightarrow$ Knowledge representation and reasoning; Supervised learning by classification; • Applied computing $\rightarrow$ Life and medical sciences;

\section{KEYWORDS}

drug-drug interaction; embeddings; dependency; abstract meaning representation; deep learning

\section{INTRODUCTION}

According to a recent study, medical error has become the third most common cause of death in the United States [27]. A literature review estimated a range of 210,000-400,000 deaths a year was associated with medical errors among hospital patients [17]. Medication error is one of the primary medical errors due to drug-drug interactions (DDIs) [12, 24, 34]. DDI is an unexpected change in a drug's effect on the human body when the drug and a second drug are co-prescribed and taken together. As a matter of fact, many such errors are preventable adverse drug events (ADEs) [30]. In one study at six hospitals, $75 \%$ of ADEs were preventable [16]. As a result, detecting DDIs has been crucial in health safety, which may dramatically reduce drug-related harms.

As noted by the Institute of Medicine, a lack of drug knowledge is one of the most frequent proximal causes of medication errors [10]. To enrich the drug knowledge, a number of drug databases, such as DrugBank ${ }^{1}$ [21], DailyMed ${ }^{2}$, Stockley's Drug Interactions [3],

\footnotetext{
${ }^{1}$ https://www.drugbank.ca/

${ }^{2}$ https://dailymed.nlm.nih.gov/
} 
National Drug File ${ }^{3}$, and WebMD ${ }^{4}$, have been initiated. However, there still exists a large amount of hidden DDI information in the vastness of the textual biomedical literature. As the volume of the published literature grows rapidly and greatly, it is challenging and time-consuming to extract DDIs manually. Therefore, automatically extracting DDI information from literature remains an important research topic.

To accelerate the research on automatic DDI extraction, the shared task challenges for DDI extraction were organized in 2011 (DDIExtraction 2011) [37] and 2013 (DDIExtraction 2013) [36]. Many participating systems utilized identical classification models, like Support Vector machines (SVMs) [9, 33, 39]. However, the performance of these systems is highly dependent on feature engineering, and a variety of features has been adopted in each of these systems.

Recently, deep learning has been widely utilized as a feature representation method since it can transform the raw data (such as the pixel values of an image or words in a textual document) into a high level representation or feature vector [22]. Word embeddings are one of the most successful cases in deep learning that can capture high level semantic properties of words. However, the standard word embeddings ignore syntax information. Dependency embeddings have been proposed. For example, Levy and Goldberg [23] proposed dependency-based embeddings (dependency embeddings) that incorporate structural syntactic contexts into the embeddings. A study by Komninos et al. [20] shows that these structural information contributes positively to sentence classifiers. However, dependency embeddings have not been utilized in DDI extraction to the best of our knowledge.

Unlike a dependency-based representation of a sentence, Abstract Meaning Representation (AMR) is a semantic formalism that expresses the logical meanings of English sentences in the form of a directed, acyclic graph [2]. AMR aims to abstract away from syntactic idiosyncrasies and attempts to capture only the core meaning of a sentence. Thus, embeddings based on AMR could encode more information from the sentence, such as semantic roles, named entities, and co-reference information, which is important for DDI extraction. However, we cannot find any study of encoding AMR into embeddings.

In this paper, we utilize dependency and AMR embeddings as features for DDI extraction. We evaluate these embeddings on DDIExtraction 2013 challenge corpus with two different classification methods: SVM and Random Forest. We compare the performance of using different embeddings in a baseline method. The experimental results show that both dependency and AMR embeddings are effective in extracting DDIs. The best performance was obtained by using word, dependency and AMR embeddings.

The paper is organized as follows. In the next section, we briefly introduce the related work of used features for DDI extraction in the literature. In Section 3, we describe the proposed methods for DDI extraction. Experiments and results are reported in Section 4, followed by discussion and conclusion in Sections 5 and 6, respectively.

\footnotetext{
${ }^{3}$ https://www.pbm.va.gov/NationalFormulary.asp

${ }^{4}$ http://www.webmd.com/
}

\section{RELATED WORK}

Since many drug interactions are frequently reported in biomedical literature, Segura et al. [36, 37] organized the DDI extraction challenge tasks in 2011 and 2013 to stimulate research in developing automatic information extraction tools for DDI extraction from literature. The datasets for DDIExtraction 2011 were composed of sentences describing DDIs from the DrugBank database and another dataset including MedLine abstracts was added to DDIExtraction 2013. In the datasets, drug entities and DDIs were annotated at the sentence level and each sentence could contain two or more drugs. There are two subtasks considered, one is to extract DDI drug pairs and the second is to classify the pairs to different DDI types. In this paper, we focused on the first task and utilized the datasets of DDIExtraction 2013.

The top systems in DDIExtraction 2013 utilized diverse features for their classifiers. The system with the highest F1 score $(0.80)$ was proposed by the FBK-irst team [9]. They used shallow linguistic and contextual features to train a binary SVM classifier. The shallow linguistic features include features generated from shallow linguistic processing, such as tokenization, sentence splitting, Part-of-Speech (PoS) tagging and lemmatization [14]. The contextual features are a set of heterogeneous features related to the context of drug entities. The second best system proposed by Rocktaschel et al. [35] investigated the usage of domain-specific features based on handcrafted rules and domain-dependent dictionaries. Bokharaeian and Diaz [7] utilized 49 features, including lexical, morphosyntactic, conjunction, verbs, negation and parse tree features, in their SVM and achieved an F1 score of 0.66. Similarly, Bobic et al. [6] also used lexical features, dependency-based features and semantic features. Björne et al.[5] used The Turku Event Extraction System (TEES), an open source relation extraction system leveraging dependency features, and achieved an F1 score of 0.60 . However, the features used in these systems require extensive human efforts to engineer and extract and are usually specific to the datasets.

Representation learning methods, inspired by deep neural network models, transform discrete symbols into representations (usually dense vectors) at higher and abstract level. Word embedding is a word representation that captures semantic and syntactic similarities between words. It has been widely utilized for a variety of tasks, such as sentence classification [42], relation classification [41], and sentiment analysis [38], since the introduction of word2vec software. For DDI extraction from biomedical literature, word embeddings have also drawn researchers' attention recently. Liu et al. [26] deployed word embeddings in a convolutional neural network (CNN) for the DDI classification task (F1 score $=0.70)$. Zhao et al. [43] utilized word embeddings in a syntax convolutional neural network (SCNN) by changing the input of word2vec to the word sequences on the shortest path in the predicate argument sentence structure to extract DDIs (F1 score $=0.78$ ). Both systems achieved promising performance. In addition to word embeddings, dependency embeddings exhibit more functional similarity than word embeddings [23] and outperform standard word embeddings for sentence classification tasks [20]. However, dependency embeddings have not been employed for DDI extraction, to the best of our knowledge. 
Linguistically, AMR is similar to the dependency structure of a sentence where both describe relations between a head and a child [40]. On the other hand, AMR generally represents function words as relations or omits them if they have no contribution to the sentence. It utilizes multi-layer linguistic analyses including PropBank frames, non-core semantic roles, coreference, named entity annotation, modality, and negation to represent the semantic structure of a sentence and abstracts away its syntax form [15]. The nodes in AMR are entities instead of words, and the edge types are much more fine-grained. Therefore, compared to the conventional dependency parsing, AMR captures deeper and more semantic meaning [25]. However, AMR parse graphs have not been utilized in training embeddings for classification tasks in the literature. Our hypothesis is that AMR-based embeddings might be more informative for DDI extraction than dependency embeddings as AMR could capture syntactic, semantic and abstractive information.

\section{METHODS}

In this section, we first elaborate the embedding methods, including word embeddings, dependency embeddings, and AMR embeddings, and then briefly introduce the classifiers utilized for DDI extraction, including SVM, and Random Forest.

\subsection{Embedding Methods}

3.1.1 Word Embeddings. We utilized skipgram model [28, 29], a popular method of learning word embeddings by predicting context words occurring in a window around a target word. Suppose that given a target word $w_{t}$ and its context word $h$, the goal of skipgram model is to maximize the log-likelihood on the training data, i.e.,

$$
J=\log P\left(h \mid w_{t}\right) .
$$

$P\left(h \mid w_{t}\right)$ is the conditional probability in the neural probabilistic language model [4], which is usually defined as:

$$
P\left(h \mid w_{t}\right)=\sigma\left(\operatorname{score}\left(w_{t}, h\right)\right)=\frac{e^{\operatorname{score}\left(w_{t}, h\right)}}{\sum_{w^{\prime} \in V} e^{\operatorname{score}\left(w^{\prime}, h\right)}}
$$

where $V$ represents the vocabulary list, $\operatorname{score}\left(w_{t}, h\right)$ the compatibility score between $w_{t}$ and $h$ and $\sigma\left(z_{j}\right)=\frac{e^{z_{j}}}{\sum_{k=1}^{K} e^{z_{k}}}, j=1,2, \ldots, K$ a softmax function that normalizes a $K$-dimensional real vector into a $K$-dimensional real vector with summation of all elements equaling to 1 . Accordingly, the log-likelihood can be written as:

$$
J=\operatorname{score}\left(w_{t}, h\right)-\log \left(\sum_{w^{\prime} \in V} e^{\operatorname{score}\left(w^{\prime}, h\right)}\right) .
$$

In order to avoid expensive computation of softmax for the whole vocabulary, negative sampling is commonly adopted in the learning algorithm. Then the objective function becomes:

$$
J^{\prime}=\sum_{w_{t}, h \in D} \log Q_{\theta}\left(D=1 \mid w_{t}, h\right)+\sum_{w_{t}, h \in D^{\prime}} \log Q_{\theta}\left(D=0 \mid w_{t}, h\right),
$$

where $D$ is the observed data, $D^{\prime}$ the unobserved data, $\theta$ the embeddings, $Q_{\theta}\left(D=1 \mid w_{t}, h\right)$ is the probability of $w_{t}$ and $h$ being observed and $Q_{\theta}\left(D=0 \mid w_{t}, h\right)$ is the probability not being observed. Since the objective function is concave, the embeddings can be learned by setting the derivative of the objective function to 0 with respect to $\theta$, i.e., $\frac{\partial}{\partial \theta} J^{\prime}=0$, and using stochastic gradient descent on the training data batches.

As shown by previous studies [18, 33], the neighboring words (i.e., contexts) of target drug entities capture repetitive expression patterns for classifying DDIs. Since the DDI is defined for a pair of drugs in a sentence, we created a feature vector $x_{w}$ for each drug entity pair by using its associated word context embeddings, i.e.,

$$
x_{w}=\frac{1}{C} \sum_{c}^{C} \theta_{c}^{e 1} \oplus \frac{1}{C^{\prime}} \sum_{c}^{C^{\prime}} \theta_{c}^{e 2}
$$

where $\theta_{c}^{e 1}$ denotes the embeddings for the context word $c$ of drug entity $e 1, \theta_{c}^{e 2}$ is the embeddings for the context word $c$ of drug entity $e 2, C$ and $C^{\prime}$ are the number of context words for drug entities $e 1$ and $e 2$, respectively, and $\oplus$ is the concatenation operator. The reason of selecting the concatenation operator is that Komninos and Manandhar [20] verified its superiority over the linear combination of embeddings.

3.1.2 Dependency Embeddings. Unlike the conventional skipgram embedding model in which the context $h$ of a target word $w_{t}$ is the words surrounding it in a window, the dependency skipgram model $[20,23]$ considers $w_{t}$ 's syntactic context (denoted as $h_{d}$ ) in the dependency graph.

Figure 1 illustrates an example showing the word contexts and dependency contexts for the sentence "Acarbose might have a potential side effect when it is given with metformin." As we can see, the dependency contexts include discrete symbols representing syntactically connected words and the dependency relations in a dependency parse graph. For example, nsubj_inv_have is a dependency context for target word "acarbose", where "inv" denotes the reverse relation. Here we only consider the dependency contexts within distance one in the graph. Algorithm 1 shows the steps for generating the dependency contextscontexts for a target word.

Training the dependency skipgram model is similar to training the conventional skipgram except that embeddings $\left(\theta_{d}\right)$ are optimized to predict the target word's dependency contexts, i.e.,

$$
\begin{aligned}
\arg \max _{\theta_{d}} J^{\prime} & =\underset{\theta_{d}}{\arg \max _{w_{t}, h_{d} \in D}} \log Q_{\theta_{d}}\left(D=1 \mid w_{t}, h_{d}\right) \\
& +\sum_{w_{t}, h_{d} \in D^{\prime}} Q_{\theta_{d}}\left(D=0 \mid w_{t}, h_{d}\right) .
\end{aligned}
$$

The dependency feature vector $x_{d}$ for a pair of drug entities can be written as:

$$
x_{d}=\frac{1}{T} \sum_{t}^{T} \theta_{t}^{e 1} \oplus \frac{1}{T^{\prime}} \sum_{t}^{T^{\prime}} \theta_{t}^{e 2}
$$

where $\theta_{t}^{e 1}$ denotes the dependency embeddings for drug entity $e 1$ 's dependency context $t$, and $T$ and $T^{\prime}$ are the number of dependency contexts for drug entities $e 1$ and $e 2$, respectively.

3.1.3 AMR Embeddings. Since AMR provides a whole-sentence semantic representation [25], it could capture long-range dependencies among constituent words in a sentence. As shown in Figure 1 , the AMR graph for the example sentence is an abstractive representation of the semantic meaning in the sentence. The key word "effect" reflecting the DDI between "acarbose" and "metformin" is an AMR context for "acarbose" (i.e., "ARG1_inv_effect") whereas 
"Acarbose may have a potential side effect when it is given with metformin."

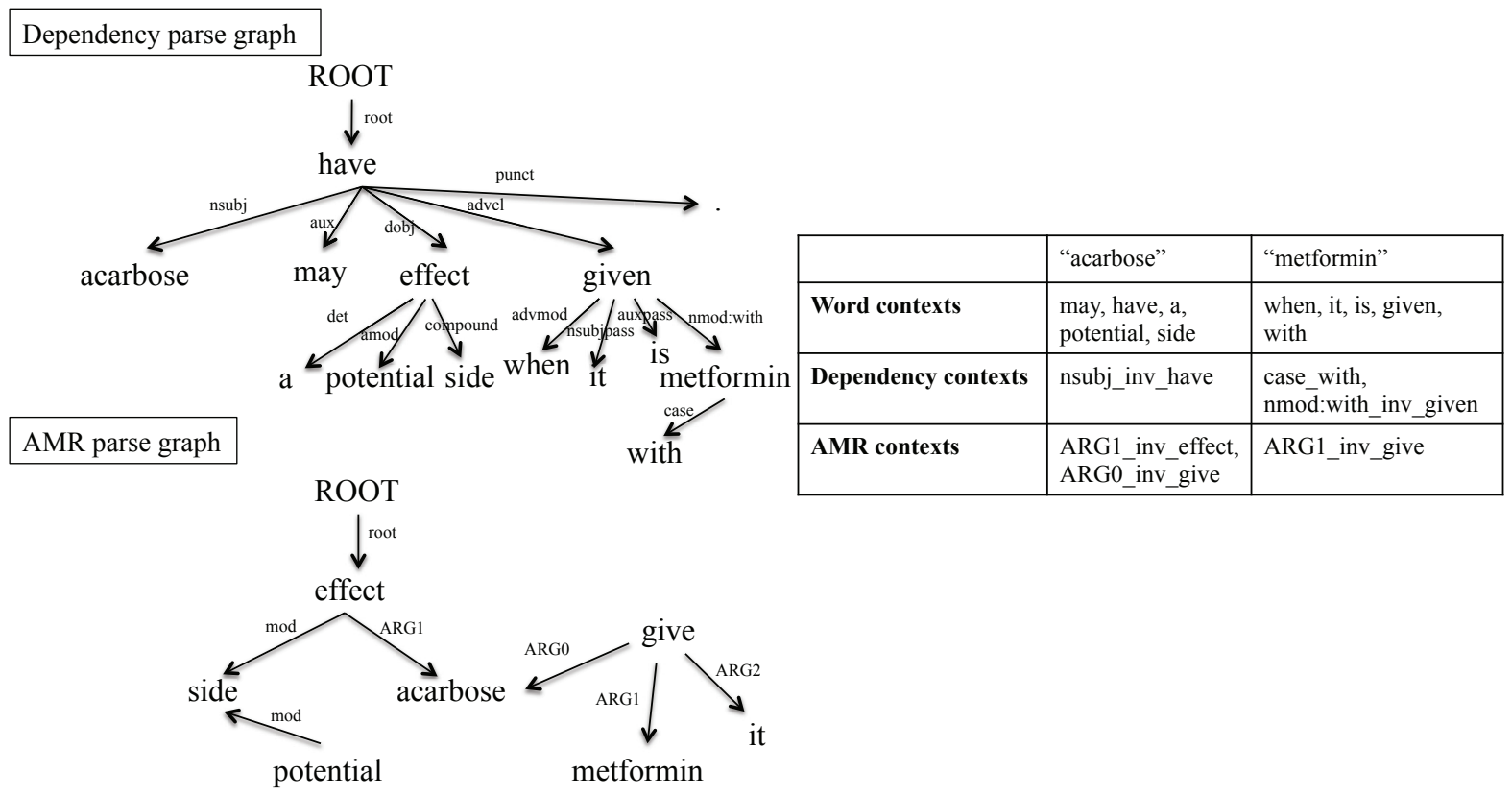

Figure 1: An example of word contexts, dependency contexts, and AMR contexts for a sentence with drug-drug interactions.

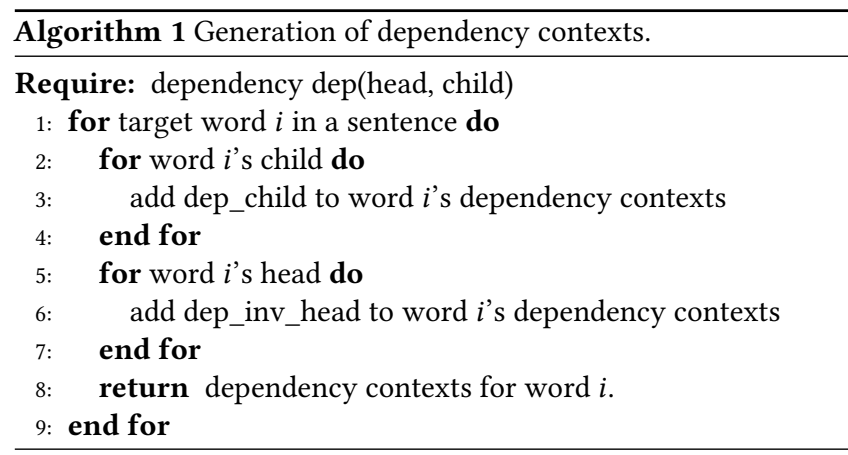

neither word contexts nor dependency contexts capture this information.

In our framework, we first parse DDI sentences into AMR graphs, generate the AMR contexts for each target word, and then input the target word and the corresponding AMR contexts into the skigram model. In the first step, we use JAMR [13], a statistical semantic parser trained on AMR bank, for AMR parsing. Similar to many other statistical parsing methods, JAMR also significantly depends on the training data [31]. Considering that many drug names are rarely appeared in the AMR bank and that the contexts of drug entities are crucial for detecting drug-drug relation instead of drug entities themselves, we replace "drug" with "medication", drug entities with "drug" and run the AMR parsing algorithm. Then, after AMR parsing we restore the drug entities and "drug". By doing so, we could parse the drug entities correctly without losing any drug information. Figure 2 shows the procedure of generating AMR contexts for the example sentence.
After obtaining the AMR contexts, we utilize the dependency skipgram model to learn the AMR embeddings and define AMR embeddings feature vector $x_{a}$ for a pair of drug entities as:

$$
x_{a}=\frac{1}{M} \sum_{m}^{M} \theta_{m}^{e 1} \oplus \frac{1}{M^{\prime}} \sum_{m}^{M^{\prime}} \theta_{m}^{e 2}
$$

where $\theta_{m}^{e 1}$ denotes the AMR embeddings for the AMR context $m$ of drug entity $e 1$, and $M$ and $M^{\prime}$ are the number of AMR contexts for drug entities $e 1$ and $e 2$, respectively.

\subsection{Classification Methods}

As mentioned in Section 2, we focus on the DDI extraction for each drug-drug pair. For a given drug pair, we use binary classifiers to detect whether the drug pair has interactions (positive) or not (negative). In this section, we elaborate two classifiers used for this DDI extraction task: SVM and Random Forest.

3.2.1 Support Vector Machines. Given the embedding feature vector $x_{i}$ of the $i$ th drug entity pair in the training data, we train a linear SVM for detecting DDIs in the testing data. We denote $y_{i}=1$ if the interaction of drug entity pair is positive and $y_{i}=-1$ otherwise. Then the cost function can be written as

$$
\min _{w, b} \frac{1}{2} w^{T} w+C \sum_{i}^{N} \max \left(1-y_{i}\left(w^{T} x_{i}+b, 0\right)\right) .
$$

For the drug pair in the testing data with embedding feature vector $\tilde{x}$, the prediction of DDI is defined by the decision function:

$$
\tilde{y}=\operatorname{sgn}\left(w^{T} \tilde{x}+b\right) \text {. }
$$




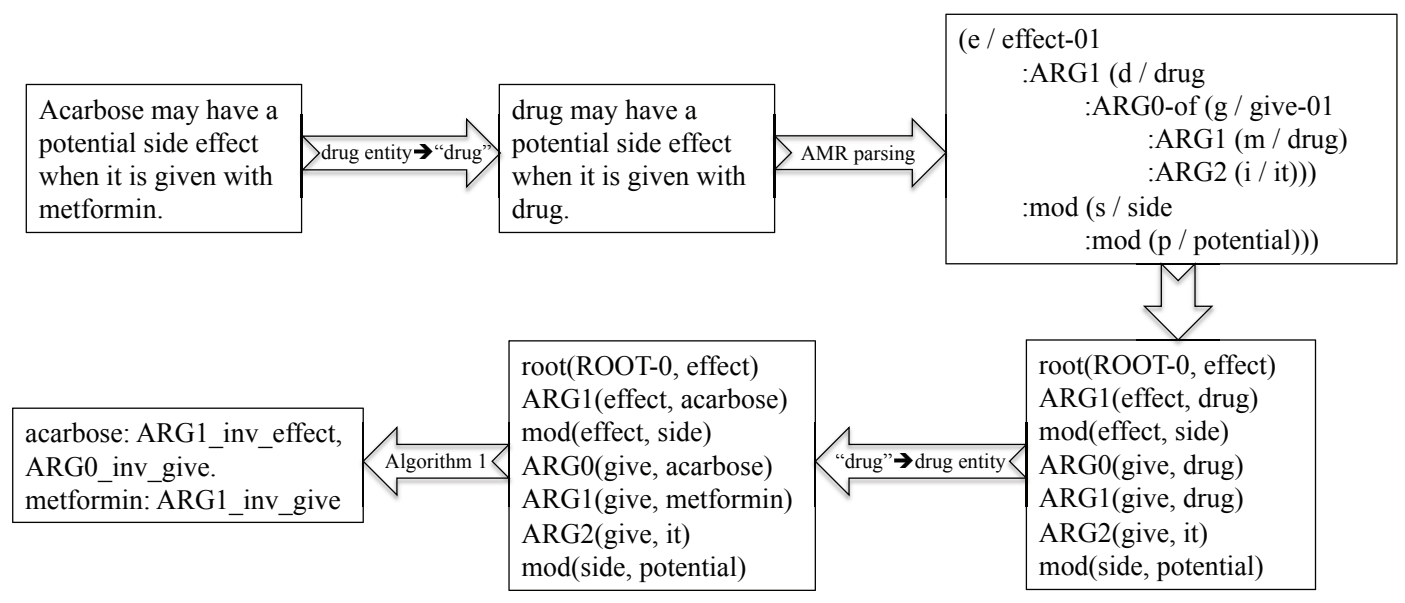

Figure 2: An example of generating AMR contexts.

3.2.2 Random Forest. Random Forest is an ensemble of classification trees, where each tree contributes with a single vote for the assignment of the most frequent class to the input data [8]. Compared to other classifiers, Random Forest usually has high classification accuracy and ability to model complex interactions among input variables. In training, Random Forest creates multiple trees by bootstrap aggregating from the original training data on each drug pair, and splits each node by searching across a randomly selected subset of the input variables. For classification of the unseen embedding feature vector $\tilde{x}$, the prediction of DDI is made by averaging the predictions from all the individual regression trees on $\tilde{x}$ :

$$
\tilde{y}=\frac{1}{B} \sum_{b=1}^{B} f_{b}(\tilde{x}) .
$$

where $B$ is the number of trees and $f_{b}$ is a trained decision tree at $b$ th step.

\section{EXPERIMENTS}

The proposed methods were evaluated on the DDI corpus provided by the DDIExtraction 2013 challenge. The corpus contains 142 MedLine abstracts on the subject of DDIs, and 572 documents from the DrugBank database. There are a total of 6976 sentences with annotated drug entities and DDIs. The corpus was randomly split by the organizer into training dataset (77\%) and testing dataset (23\%). $17.2 \%$ of all possible drug pairs are positive pairs, while the rest are negatives. In our problem setting, we assume that all drug entities are provided. Our goal is to detect whether a given drug pair in a sentence has interactions or not. We trained the embeddings on the entire corpus. Using labels in the training dataset and embedding features, we trained the classifiers with 10 -fold cross-validation and then evaluated the methods on the testing dataset.

The features used in the baseline method include words and word bigrams with binary values indicating their presence or absence in a sentence, cosine similarity between centroid vector of each class and the instance, negation (three features indicating negation before the first main drug, between two main drugs, and after the two main drugs), and mutual information. Detailed information about these features can be found in [33] and [32].

Let the baseline feature vector for a drug entity pair be $x_{0}$. For the same drug entity pair, we calculate word embeddings $x_{w}$, dependency embeddings $x_{d}$, and AMR embeddings $x_{a}$ according to Equations 5, 7 and 8, respectively. We compare the results using Baseline (i.e., $x_{0}$ ), Baseline + WordEmbeddings (i.e., $x_{0} \oplus x_{w}$ ), Baseline + DepEmbeddings (i.e., $x_{0} \oplus x_{d}$ ), Baseline + AMREmbeddings (i.e., $x_{0} \oplus x_{a}$ ), Baseline + WordEmbeddings + DepEmbeddings (i.e., $\left.x_{0} \oplus x_{w} \oplus x_{d}\right)$, Baseline + WordEmbeddings + DepEmbeddings + AMREmbeddings (i.e., $x_{0} \oplus x_{w} \oplus x_{d} \oplus x_{a}$ ). A DDI is considered to be correctly detected only if the system is able to assign the correct prediction label (positive vs. negative) to the candidate drug entity pair. The evaluation is drug-drug relation oriented and based on the micro-average F1 score of both classes.

Appropriate preprocessing could enhance the final performance. Since numbers (digits) occurred frequently in the corpus, we replaced all the digits with " 7 ". Then all tokens were converted to lowercase. For AMR parsing, an additional preprocessing step is the replacement of drug entities with "drug", as shown in Figure 2, due to the fact that many drug names are not in the AMR vocabulary. Note that we used a special symbol to replace "drug" if it appears in the original text before this additional preprocessing and restored "drug" after AMR parsing.

Stanford CoreNLP ${ }^{5}$ tools were employed to obtain dependencies and $\mathrm{JAMR}^{6}$ was used for AMR parsing. We utilized the modified word2vec implementation [20] to generate the dependency and AMR embeddings. The negative-sampling parameter was set to 15 , and the dimension of embeddings was set to 100 . The machine learning library Scikit-learn ${ }^{7}$ 0.18.1 was used for the binary label classification of positive and negative DDI pairs. For SVM, we tried a series of $C$ values, and utilized the optimal value $C=0.10$. For Random Forest, the number of estimators was tuned and set at the optimal value of 40 .

\footnotetext{
${ }^{5}$ https://stanfordnlp.github.io/CoreNLP/

${ }^{6}$ https://github.com/jflanigan/jamr

${ }^{7}$ http://scikit-learn.org/stable/index.html
} 
Table 1: F1 score on the DDIExtraction 2013 test dataset.

\begin{tabular}{|c|c|c|c|c|c|c|}
\hline \multirow[b]{2}{*}{ Embeddings } & \multicolumn{2}{|c|}{ DrugBank (5265 pairs) } & \multicolumn{2}{|c|}{ MedLine (451 pairs) } & \multicolumn{2}{|c|}{ Total (5716 pairs) } \\
\hline & SVM & Random Forest & SVM & Random Forest & SVM & Random Forest \\
\hline Baseline & 0.66 & 0.59 & 0.69 & 0.69 & 0.74 & 0.76 \\
\hline Baseline+WordEmbeddings & $0.73(+11 \%)$ & $0.84(+42 \%)$ & $0.68(-1 \%)$ & $0.70(+1 \%)$ & $0.77(+4 \%)$ & $0.81(+7 \%)$ \\
\hline Baseline+DepEmbeddings & $0.68(+3 \%)$ & $0.84(+42 \%)$ & $0.62(-10 \%)$ & $0.72(+4 \%)$ & $0.77(+4 \%)$ & $0.83(+9 \%)$ \\
\hline Baseline+AMREmbeddings & $0.64(-3 \%)$ & $0.82(+39 \%)$ & $0.67(-3 \%)$ & $0.70(+1 \%)$ & $0.77(+4 \%)$ & $0.82(+8 \%)$ \\
\hline Baseline+WordEmbeddings+DepEmbeddings & $0.74(+12 \%)$ & $0.85(+44 \%)$ & $0.69(+0 \%)$ & $0.72(+4 \%)$ & $0.79(+7 \%)$ & $0.83(+9 \%)$ \\
\hline Baseline+WordEmbeddings+DepEmbeddings + AMREmbeddings & $0.75(+14 \%)$ & $0.86(+46 \%)$ & $0.69(+0 \%)$ & $0.72(+4 \%)$ & $0.78(+5 \%)$ & $\mathbf{0 . 8 4}(+11 \%)$ \\
\hline Kim et al. [18] & \multicolumn{2}{|c|}{0.80} & \multicolumn{2}{|r|}{0.47} & \multicolumn{2}{|r|}{0.78} \\
\hline FBK-irst [9] & \multicolumn{2}{|c|}{0.83} & \multicolumn{2}{|r|}{0.53} & \multicolumn{2}{|r|}{0.80} \\
\hline SCNN [43] & \multicolumn{2}{|r|}{-} & \multicolumn{2}{|r|}{ - } & \multicolumn{2}{|r|}{0.78} \\
\hline
\end{tabular}

The F1 scores of methods are listed in Table 1. As shown in the table, the best performance measures are achieved by Random Forest utilizing baseline and three embedding features together with an F1 score of 0.86 for DrugBank, 0.72 for MedLine, and 0.84 overall. We observe that adding any of three embedding features improves the baseline performance. For DrugBank, Baseline + DepEmbeddings is comparative with Baseline + WordEmbeddings and superior to Baseline + AMREmbeddings when Random Forest classifier is used. For MedLine, Baseline + DepEmbeddings performs the best when only one embedding feature is considered. Though the results of Baseline + AMREmbeddings are similar to those of Baseline + WordEmbeddings and inferior to those of Baseline + DepEmbeddings, adding AMR embeddings always improves the performance when Random Forest classifier is used. It means that AMR embeddings contain alternative useful information for DDI extraction. Compared with the state-of-the-art systems, including the best existing system (FBK-irst [9]) and CNN-based system (SCNN [43]), the proposed method shows better performance.

\section{DISCUSSION}

Our experiment shows that dependency and AMR embeddings provide valuable information for DDI extraction thus the performance improves when they are added to the baseline. This result indicates that dependency and AMR embeddings should be utilized in conjunction with the word embeddings to obtain the best performance. This is consistent with previous study [20], where syntactic contexts were combined with word contexts for sentence classification.

The results using AMR embeddings alone are not promising as we expected. This might be due to that the pre-trained model we used for JAMR -LDC2014T12 [19]- does not contain sufficient medical terms and documents. Figure 3 shows an example that was misclassified by AMR embeddings. In the sentence "Consequently, the combination of methotrexate with acitretin is also contraindicated.", the word "contraindicated" plays an important role in determining the interaction between "methotrexate" and "acitretin". Since the pre-trained model for AMR parsing does not contain this term, this information could not be captured by AME embeddings. Other misclassified cases are due to the limitations of AMR. For example, AMR does not deeply capture noun-noun or noun-adjective relations [1].
"Consequently, the combination of methotrexate with acitretin is also contraindicated."

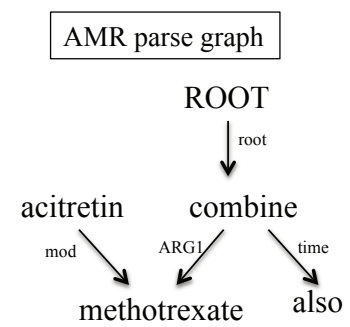

Figure 3: A misclassified example by AMR embeddings.

A limitation associated with both dependency and AMR embeddings is that only contexts within distance one in the dependency or AMR graph are considered. Increasing the distance might include more information in the embeddings but Algorithm 1 should be refined to avoid adding redundant contexts. In addition, another option is to add the nodes at the same level to incorporate more significant words for detecting DDIs. In Figure 1, "effect" would be added if such strategy was taken. However, this strategy will also add redundant contexts. For example, "when", "it" and "may" will also be considered as contexts for the example in Figure 1. Thus, more sophisticated methods are needed to study what are the best contexts in the graph for learning dependency and AMR embeddings.

We observe that Random Forest performs better than SVM except the DrugBank dataset. The reason is that Random Forest could provide a more complex model when dimensionality is less than the sample size. The study conducted by Delgado et al. tested 179 classifiers and found that Random Forest was the best family of classifiers [11]. Our experiments verified this result.

We notice that the proposed methods failed for the sentences that contain multiple drug pairs. The sentence "Before taking this medication, tell your doctor if you are taking a tricyclic antidepressant such as amitriptyline (Elavil), amoxapine (Asendin), doxepin (Sinequan), nortriptyline (Pamelor), imipramine (Tofranil), clomipramine (Anafranil), protriptyline (Vivactil), or desipramine 
(Norpramin)." contains 16 drugs and 136 drug pairs. The reason that the dependency embeddings failed to detect these negative DDIs might be that each drug pair was connected by conjunct relation in the dependency graph. Another misclassified example is "Single dose pharmacokinetic studies demonstrated that the metabolism of rivastigmine is not significantly affected by concurrent administration of digoxin, warfarin, diazepam, or fluoxetine.", which consists of 4 drugs and 10 drug pairs. The proposed methods failed to identify the negated interaction between "rivastigmine" and any of "warfarin", "diazepam", and "fluoxetine".

\section{CONCLUSION AND FUTURE WORK}

In this paper, we proposed dependency embeddings and novel AMR embeddings for detecting DDIs from biomedical literature. We evaluated the proposed methods on DDIExtraction-2013 corpus with two different classification methods. The experiments shows the effectiveness of dependency embeddings and AMR embeddings. The best performance was obtained by using word, dependency and AMR embeddings in the baseline.

In the future work, we would like to utilize more comprehensive classifiers, such as Convolutional Neural Network (CNN) and Long Short Term Memory network (LSTM), for detecting DDIs. Since the dimension of embeddings might impact the classification, we also want to investigate what the best dimension is for the DDI extraction task. Moreover, extending the proposed methods to detect undiscovered DDIs from a large number of biomedical articles is subject to a future study.

\section{ACKNOWLEDGMENTS}

This work has been supported by the National Institute of Health (NIH) grants R01LM011934 and R01GM102282.

\section{REFERENCES}

[1] Laura Banarescu, Claire Bonial, Shu Cai, Madalina Georgescu, Kira Griffitt, Ulf Hermjakob, Kevin Knight, Philipp Koehn, Martha Palmer, and Nathan Schneider 2012. Abstract meaning representation (AMR) 1.2 specification. In Parsing on Freebase from Question-Answer Pairs. In Proceedings of the 2013 Conference on Empirical Methods in Natural Language Processing. Seattle: ACL. 1533-1544.

[2] Laura Banarescu, Claire Bonial, Shu Cai, Madalina Georgescu, Kira Griffitt, Ulf Hermjakob, Kevin Knight, Philipp Koehn, Martha Palmer, and Nathan Schneider. 2013. Abstract Meaning Representation for Sembanking. In Proceedings of the 7th Linguistic Annotation Workshop \& Interoperability with Discourse. 178-186.

[3] Karen Baxter and Claire L Preston. 2010. Stockley's drug interactions. Vol. 495 Pharmaceutical Press London.

[4] Yoshua Bengio, Réjean Ducharme, Pascal Vincent, and Christian Jauvin. 2003. A neural probabilistic language model. fournal of machine learning research 3, Feb (2003), 1137-1155.

[5] Jari Björne, Suwisa Kaewphan, and Tapio Salakoski. 2013. UTurku: drug named entity recognition and drug-drug interaction extraction using SVM classification and domain knowledge. In Second foint Conference on Lexical and Computational Semantics $\left({ }^{*}\right.$ SEM), Vol. 2. 651-659.

[6] Tamara Bobic, Juliane Fluck, and Martin Hofmann-Apitius. 2013. SCAI: Extracting drug-drug interactions using a rich feature vector. In Second foint Conference on Lexical and Computational Semantics. Citeseer, 675

[7] Behrouz Bokharaeian and Alberto Díaz. 2013. NIL UCM: Extracting Drug-Drug interactions from text through combination of sequence and tree kernels. In Second foint Conference on Lexical and Computational Semantics. 644-650.

[8] Leo Breiman. 2001. Random forests. Machine learning 45, 1 (2001), 5-32.

[9] Md Faisal Mahbub Chowdhury and Alberto Lavelli. 2013. FBK-irst: A multi-phase kernel based approach for drug-drug interaction detection and classification that exploits linguistic information. Atlanta, Georgia, USA 351 (2013), 53.

[10] Linda R Cronenwett, J Lyle Bootman, Julie Wolcott, Philip Aspden, and others 2007. Preventing medication errors: quality chasm series. National Academies Press.
[11] Manuel Fernández-Delgado, Eva Cernadas, Senén Barro, and Dinani Amorim. 2014. Do we need hundreds of classifiers to solve real world classification problems. F. Mach. Learn. Res 15, 1 (2014), 3133-3181.

[12] Noha Ferrah, Janaka J Lovell, and Joseph E Ibrahim. 2017. Systematic review of the prevalence of medication errors resulting in hospitalization and death of nursing home residents. Journal of the American Geriatrics Society 65, 2 (2017), 433-442.

[13] Jeffrey Flanigan, Sam Thomson, Jaime G Carbonell, Chris Dyer, and Noah A Smith. 2014. A discriminative graph-based parser for the abstract meaning representation. (2014).

[14] Claudio Giuliano, Alberto Lavelli, and Lorenza Romano. 2006. Exploiting shallow linguistic information for relation extraction from biomedical literature.. In $E A C L$, Vol. 18. Citeseer, 401-408.

[15] Lifu Huang, Jonathan May, Xiaoman Pan, and Heng Ji. 2016. Building a FineGrained Entity Typing System Overnight for a New X (X= Language, Domain, Genre). arXiv preprint arXiv:1603.03112 (2016).

[16] Balthasar L Hug, Daniel J Witkowski, Colin M Sox, Carol A Keohane, Diane L Seger, Catherine Yoon, Michael E Matheny, and David W Bates. 2010. Adverse drug event rates in six community hospitals and the potential impact of computerized physician order entry for prevention. Fournal of general internal medicine 25,1 (2010), 31-38.

[17] John T James. 2013. A new, evidence-based estimate of patient harms associated with hospital care. fournal of patient safety 9, 3 (2013), 122-128.

[18] Sun Kim, Haibin Liu, Lana Yeganova, and W John Wilbur. 2015. Extracting drug-drug interactions from literature using a rich feature-based linear kernel approach. Journal of biomedical informatics 55 (2015), 23-30.

[19] Kevin Knight, Lauren Baranescu, Claire Bonial, Madalina Georgescu, Kira Griffitt, Ulf Hermjakob, Daniel Marcu, Martha Palmer, and Nathan Schneifer. 2014. Abstract meaning representation (AMR) annotation release 1.0. Web download (2014).

[20] Alexandros Komninos and Suresh Manandhar. 2016. Dependency based embeddings for sentence classification tasks. In Proceedings of NAACL-HLT. 1490-1500.

[21] Vivian Law, Craig Knox, Yannick Djoumbou, Tim Jewison, An Chi Guo, Yifeng Liu, Adam Maciejewski, David Arndt, Michael Wilson, Vanessa Neveu, and others. 2014. DrugBank 4.0: shedding new light on drug metabolism. Nucleic acids research 42, D1 (2014), D1091-D1097.

[22] Yann LeCun, Yoshua Bengio, and Geoffrey Hinton. 2015. Deep learning. Nature 521, 7553 (2015), 436-444.

[23] Omer Levy and Yoav Goldberg. 2014. Dependency-Based Word Embeddings.. In ACL (2). Citeseer, 302-308.

[24] Dingcheng Li, Sijia Liu, Majid Rastegar-Mojarad, Yanshan Wang, Vipin Chaudhary, Terry Therneau, and Hongfang Liu. 2016. A Topic-modeling Based Framework for Drug-drug Interaction Classification from Biomedical Text. In AMIA Annual Symposium Proceedings, Vol. 2016. American Medical Informatics Association, 789 .

[25] Fei Liu, Jeffrey Flanigan, Sam Thomson, Norman Sadeh, and Noah A Smith. 2015. Toward abstractive summarization using semantic representations. In Proceedings of the North American Chapter of the Association for Computational Linguistics (NAACL).

[26] Shengyu Liu, Buzhou Tang, Qingcai Chen, and Xiaolong Wang. 2016. Drug-drug interaction extraction via convolutional neural networks. Computational and mathematical methods in medicine 2016 (2016).

[27] Martin A Makary and Michael Daniel. 2016. Medical error?the third leading cause of death in the US. Bmj 353 (2016), i2139.

[28] Tomas Mikolov, Kai Chen, Greg Corrado, and Jeffrey Dean. 2013. Efficient estimation of word representations in vector space. arXiv preprint arXiv:1301.3781 (2013).

[29] Tomas Mikolov, Ilya Sutskever, Kai Chen, Greg S Corrado, and Jeff Dean. 2013. Distributed representations of words and phrases and their compositionality. In Advances in neural information processing systems. 3111-3119.

[30] Teryl K Nuckols, Crystal Smith-Spangler, Sally C Morton, Steven M Asch, Vaspaan M Patel, Laura J Anderson, Emily L Deichsel, and Paul G Shekelle. 2014. The effectiveness of computerized order entry at reducing preventable adverse drug events and medication errors in hospital settings: a systematic review and meta-analysis. Systematic reviews 3, 1 (2014), 56.

[31] Xiaochang Peng, Chuan Wang, Daniel Gildea, and Nianwen Xue. 2017. Addressing the Data Sparsity Issue in Neural AMR Parsing. arXiv preprint arXiv:1702.05053 (2017).

[32] Majid Rastegar-Mojarad. 2013. Extraction and Classification of Drug-Drug Interaction from Biomedical Text Using a Two-Stage Classifier. Theses and Dissertations 304 (2013).

[33] Majid Rastegar-Mojarad, Richard D Boyce, and Rashmi Prasad. 2013. UWMTRIADS: classifying drug-drug interactions with two-stage SVM and postprocessing. In Proceedings of the 7th International Workshop on Semantic Evaluation. 667-674.

[34] Nancy E Reame, Tammy L Wyman, David J Phillips, David M de Kretser, and Vasantha Padmanabhan. 1998. Net Increase in Stimulatory Input Resulting from a Decrease in Inhibin B and an Increase in Activin A May Contribute in Part to the 
Rise in Follicular Phase Follicle-Stimulating Hormone of Aging Cycling Women 1. The fournal of Clinical Endocrinology \& Metabolism 83, 9 (1998), 3302-3307.

[35] Tim Rocktäschel, Torsten Huber, Michael Weidlich, and Ulf Leser. 2013. WBINER: The impact of domain-specific features on the performance of identifying and classifying mentions of drugs. In Second foint Conference on Lexical and Computational Semantics, Vol. 2. 356-363.

[36] Isabel Segura Bedmar, Paloma Martínez, and María Herrero Zazo. 2013. Semeval2013 task 9: Extraction of drug-drug interactions from biomedical texts (ddiextraction 2013). Association for Computational Linguistics.

[37] Isabel Segura Bedmar, Paloma Martinez, and Daniel Sánchez Cisneros. 2011. The 1st DDIExtraction-2011 challenge task: Extraction of Drug-Drug Interactions from biomedical texts. (2011).

[38] Duyu Tang, Furu Wei, Nan Yang, Ming Zhou, Ting Liu, and Bing Qin. 2014. Learning Sentiment-Specific Word Embedding for Twitter Sentiment Classification. In ACL (1). 1555-1565.
[39] Philippe Thomas, Mariana Neves, Tim Rocktäschel, and Ulf Leser. 2013. WBIDDI: drug-drug interaction extraction using majority voting. In Second foint Conference on Lexical and Computational Semantics ( ${ }^{*}$ SEM), Vol. 2. 628-635.

[40] Chuan Wang, Nianwen Xue, Sameer Pradhan, and Sameer Pradhan. 2015. A Transition-based Algorithm for AMR Parsing.. In HLT-NAACL. 366-375.

[41] Daojian Zeng, Kang Liu, Siwei Lai, Guangyou Zhou, Jun Zhao, and others. 2014. Relation Classification via Convolutional Deep Neural Network.. In COLING. 2335-2344.

[42] Xiang Zhang, Junbo Zhao, and Yann LeCun. 2015. Character-level convolutional networks for text classification. In Advances in neural information processing systems. 649-657.

[43] Zhehuan Zhao, Zhihao Yang, Ling Luo, Hongfei Lin, and Jian Wang. 2016. Drug drug interaction extraction from biomedical literature using syntax convolutional neural network. Bioinformatics 32, 22 (2016), 3444-3453. 